\title{
Use of Palliative Care Consultation for Patients with End-Stage Liver Disease: Survey of Liver Transplant Service Providers
}

\author{
Kendall R. Beck, MD, ${ }^{1}$ Steven Z. Pantilat, MD, David L. O’Riordan, PhD, and Marion G. Peters, MD ${ }^{1}$
}

\begin{abstract}
Background/Aim: Palliative care services (PCS) are recommended to enhance quality of care for hospitalized patients.

Methods: We evaluated the attitudes of liver transplant (LT) providers and perceived barriers to PCS for their patients by conducting a web-based survey of intensive care unit nurses, postgraduate year 1 (PGY1) physician trainees, nurse practitioners, fellows, and attending physicians on the LT service at an academic medical center. Results: The response rate was 44\% (88/200). Providers agreed that LT and PCS are not mutually exclusive $(86 \%, n=76)$. Respondents reported confusion regarding criteria and timing for referral to PCS. Most suggested that referral is appropriate when death is imminent $(78 \%, n=69)$. Many providers felt that patients' depression $(66 \%, n=58)$ was poorly managed, although few identified that PCS were consulted for depression $(28 \%$, $n=25)$. Overall, $84 \%(n=74)$ identified attending physicians as the main barrier to involving PCS, and attendings $(93 \%, n=82)$ were more likely than PGY1 $(67 \%, n=59)$ and nurses $(55 \%, n=48)$ to describe PCS as end-of-life care $(p=0.03)$. Nearly all LT providers agreed that patients welcomed goals of care discussions $(83 \%, n=73)$, were grateful for PCS $(96 \%, n=85)$, and received higher quality care with PCS $(96 \%, n=85)$. Conclusion: LT providers overwhelmingly report that PCS benefit patients and are consistent with LT goals even while patients are listed for LT. Barriers to PCS include confusion over referral criteria and describing PCS as end-of-life care by attending physicians. PCS teams may expand access for LT patients by establishing clear criteria for PCS referral and targeting educational interventions about palliative care to attendings.
\end{abstract}

\section{Introduction}

$\mathbf{E}$ ND-STAGE LIVER disease (ESLD) is a major cause of morbidity and mortality worldwide. Liver transplant (LT) offers a cure to thousands of patients each year; however, due to strict criteria, many patients are not candidates for transplant, and another subset die while awaiting transplant due to lack of donor or liver decompensation. According to the Scientific Registry of Transplant Recipients/ Organ Procurement and Transplantation Network 2012 report, in the United States in 2011 15,428 patients were listed for LT and only 5660 transplants were performed. Of the listed patients, 2187 patients died while active on the list, and 815 were removed from the list because they were too sick to undergo transplant. ${ }^{1}$
Patients with ESLD carry a predicted three-month mortality of greater than $50 \%$ once their Model for End-Stage Liver Disease (MELD) score exceeds $30 .^{2}$ Furthermore, they have high readmission rates, with one study finding that $69 \%$ of patients with decompensated cirrhosis were readmitted to the hospital at least once with a median time to readmission of 67 days, and $14 \%$ were readmitted within one week of discharge. ${ }^{3}$ In addition to the many life-threatening complications of ESLD, such as hepatocellular carcinoma, variceal hemorrhage, and spontaneous bacterial peritonitis, patients experience a multitude of symptoms that are difficult to control and negatively affect their quality of life. Some of these symptoms include pain, nausea, depression, cachexia, fatigue, and pruritus. ${ }^{4}$

Palliative care (PC) represents a comprehensive model of care providing symptom management, advance care planning,

\footnotetext{
${ }^{1}$ Division of Gastroenterology, Department of Medicine, University of California San Francisco, San Francisco, California.

${ }^{2}$ Palliative Care Program, Division of Hospital Medicine, Department of Medicine, University of California San Francisco, San Francisco, California.

Accepted March 17, 2016.
} 
and psychosocial and spiritual support throughout serious illness and at the end of life. PC has been shown to improve symptom management, increase quality of life, reduce depression, improve satisfaction, and decrease resource utilization for people with a variety of serious illnesses. ${ }^{5,6}$ In a randomized trial of PC for people with newly diagnosed metastatic lung cancer, PC was found to reduce pain and depression, improve quality of life, and extend life by 2.7 months. ${ }^{7}$ In other studies, the caregivers of people who received PC were less likely to have depression or complicated grief six months after their loved one died. ${ }^{8}$ Given the high morbidity and mortality of ESLD, regardless of listing status, and the high rates of hospitalization, these patients would likely benefit from a PC consultation. ${ }^{9}$ Despite a high incidence of pain or nausea, few patients delisted from the LT list were referred for PC consultation (10\% of cases). ${ }^{10}$ Little is known about the attitudes of LT providers toward palliative care services (PCS) for their patients or what the barriers are to PCS consultation. We conducted a survey of LT clinicians to determine the attitudes of the providers toward PC consultation for ESLD patients awaiting liver transplantation and to identify any potential perceived barriers toward involving PC in these very ill patients. We hypothesized that LT providers believed they are effectively managing their patients' symptoms such as pain and nausea. We also hypothesized that LT providers believed that LT listing and PC consultation were mutually exclusive, and that patients and their families would not welcome PC consultation, and that patients would perceive the LT team as giving up hope.

\section{Methods}

\section{Subjects}

Participants from a list of LT providers were asked to be involved in the survey. Respondents were categorized as follows: "Nurses" were registered nurses working in the intensive care unit, "PGY1" included postgraduate year 1 medical and surgical trainees as well as nurse practitioners (because they function in similar capacities on the LT service), and "Attendings" included gastroenterology and advanced hepatology fellows, hepatology attending physicians, as well as transplant surgical fellows and transplant attending surgeons.

\section{Survey}

The survey was designed to assess potential barriers to involving PCS in the care of ESLD patients being cared for by the LT service; to assess the LT clinicians' perceived quality of palliative symptom management; and to identify opportunities or barriers toward involving PCS in the care of the LT population. Basic demographic information collected from respondents included age, gender, clinical role, and LT experience. Survey items examined LT providers' attitude toward the management of symptoms such as pain, nausea, and depression, their documentation of patients' goals of care (GOC), their perception of patients' receptiveness to discussing GOC, beliefs on when GOC should be discussed and which provider should lead those discussions (primary care physician versus hepatologist), benefits of PC consultation, and potential barriers of involving the PCS in the care of hospitalized LT patients. Survey items were developed using an iterative process involving researchers with extensive clinical experience in the field of hepatology (M.G.P.) and PC (S.Z.P.). This experience also aided in providing face and content validity of the survey items. ${ }^{11}$ Participants responded to survey items using a four- or five-point Likert scale as necessary.

\section{Procedure}

The survey was conducted between October 2012 and January 2013. Eligible participants included all physicians, nurse practitioners, and intensive care unit nurses who cared for hospitalized LT patients over a one-year period (July 2011 to June 2012) at an academic center LT unit. Potential subjects were identified by service schedules, recruited by email, and those agreeing to participate provided informed consent via e-mail to the study coordinator. They then completed a survey using a secure, web-based data capture tool. No monetary incentives were provided, and three reminder e-mails were sent before participants were considered nonresponsive. The study protocol conformed to the ethical guidelines of the 1975 Declaration of Helsinki as reflected in an a priori approval by the UCSF Committee on Human Research.

\section{Statistical analysis}

Data were downloaded directly from the online survey software into Excel spreadsheets and subsequently exported the Statistical Package for the Social Sciences (SPSS) for Mac (version 22), which was used to analyze these data. ${ }^{12}$ Due to our small sample size and to assist with ease of interpretation, questions with response categories "Strongly Agree," "Agree," "Disagree," and "Strongly Disagree" were dichotomized to "Agree" (Strongly Agree/Agree) and "Disagree" (Disagree/Strongly Disagree). Questions with response categories "Always," "Usually," "Sometimes," "Rarely," and "Never" were dichotomized to "Always" (Always/Usually) and "Sometimes" (Sometimes/Rarely/Never). Descriptive statistics, including mean, standard deviation, and percentages, were calculated as appropriate. Chi-squared $\left(\chi^{2}\right)$ analysis was used to test for bivariate associations between categorical variables to examine associations between categorical variables. An $\alpha$ value of 0.05 was used to establish statistical significance. $p$ Values represent significance across all three variables (i.e., attendings, nurses, and PGY1).

\section{Results}

\section{Participant characteristics}

Overall, our survey was completed by 88 of the 200 LT clinicians, resulting in a $44 \%$ response rate. Respondents consisted of 53\% intensive care unit nurses, 26\% PGY1 (19 medical interns, 1 surgical intern, and 3 nurse practitioners), and $21 \%$ attendings (6 gastroenterology or advanced hepatology fellows, 1 surgical fellow, 7 hepatology attendings, and 4 surgical attendings). Pertinent demographics are reported in Table 1. During the study period, there were 614 LT patients admitted to the hospital, with 39 deaths in the hospital. Only $2 \%(n=29)$ were referred to PCS, of whom 19 (65\%) died in the hospital. Overall, 49\% (19/39) of the LT patients who died in the hospital were also cared for by the PCS. 
Table 1. Characteristics of Liver Transplant Unit Respondents to Palliative Care Survey

\begin{tabular}{lcccc}
\hline Characteristic & Nurses $(\mathrm{n}=46), \%(\mathrm{n})$ & $P G Y 1(\mathrm{n}=23), \%(\mathrm{n})$ & Attendings $(\mathrm{n}=18), \%(\mathrm{n})$ & Total $(\mathrm{n}=88), \%(\mathrm{n})$ \\
\hline $\begin{array}{l}\text { Gender } \\
\text { Male }\end{array}$ & $20(9)$ & $26(6)$ & $44(8)$ & $25(22)$ \\
Age (years) & $7(4)$ & $35(8)$ & & \\
$\quad<30$ & $46(21)$ & $57(13)$ & $61(11)$ & $14(12)$ \\
$30-39$ & $30(14)$ & $9(2)$ & $17(3)$ & $54(47)$ \\
$40-49$ & $13(6)$ & 0 & $22(4)$ & $21(18)$ \\
$>50$ & & & $0(1)$ \\
Years LT experience & 0 & $48(11)$ & $17(3)$ & $13(11)$ \\
$<1$ & $7(4)$ & $35(8)$ & $11(2)$ & $17(15)$ \\
$1-3$ & $20(9)$ & $7(2)$ & $72(13)$ & $15(13)$ \\
$4-5$ & $72(33)$ & $7(2)$ & $56(49)$ \\
$>6$ & & & \\
\hline
\end{tabular}

LT, liver transplant; PGY1, postgraduate year 1.

\section{Symptom management}

Table 2 describes respondents' perceptions of the quality of symptom management on the LT service. Specifically, regarding depression, nurses (21\%) and PGY1 (23\%) were less likely than attendings (59\%) to agree that LT providers were proficient in identifying depression $(p=0.01)$. While the minority thought that the LT service managed depression or pain well when identified in their patients, more attendings reported better management of these issues, although this did not reach statistical significance $(p=0.09$ and $p=0.07$, respectively). PGY1 trainees (86\%) and attendings (100\%) were also more likely to avoid opioid medications to treat pain out of concern that the medication would precipitate hepatic encephalopathy or precipitate removal from the LT list compared to nurses $(62 \%)(p=0.0001)$.

\section{Goals of care discussions and timing of palliative care consultation}

When respondents were asked about the appropriate time to have GOC discussions with their ESLD patients, nearly all nurses $(96 \%)$ and PGY1 $(91 \%)$ responded that it was appropriate to hold GOC discussions during clinic visits with the patient's primary care provider, while only one-third of attendings $(31 \%)$ agreed $(p=0.0001)$. In contrast, more attendings $(69 \%)$ thought it was appropriate for a patient's hepatologist to have GOC discussions during clinic visits, with $100 \%$ of nurses and $91 \%$ of PGY1 agreeing. When re- spondents were asked about GOC documentation, very few respondents reported clear documentation of GOC before hospital admission, and clinicians often had to discuss GOC with surrogate decision makers due to the development of encephalopathy in patients.

When study subjects were asked about timing of PC consultation, nurses $(88 \%)$ and PGY1 $(63 \%)$ were more likely than attendings $(44 \%)$ to report that there were patients for whom they wished they had consulted PCS earlier $(p=0.001)$. Attendings were less likely than nurses or PGY1 to agree that it is appropriate to consult PCS when the patient is diagnosed with ESLD, when the patient is listed for transplant, or when a patient with ESLD is admitted to the hospital for any reason. In contrast, the majority of attendings agreed that it is appropriate to consult PCS when the patient is imminently dying. In addition, while over $90 \%$ of nurses and PGY1 reported that there were patients who did not receive PCS consultation and whom they thought would have benefitted from one, only $50 \%$ of attendings felt the same way $(p=0.0001)$.

\section{Barriers and benefits to palliative care consultation}

Table 3 highlights barriers and benefits to obtaining PC consultation on the LT service as perceived by providers. Specifically, attending physicians, more than any other group, were identified by all respondents, including attendings, as creating a barrier to involving PCS in the care of their

Table 2. Perceived Success of Symptom Management by Liver Transplant Service

\begin{tabular}{|c|c|c|c|}
\hline Nurses $(n=47), \%(\mathrm{n})$ & $P G Y 1(n=23), \%(\mathrm{n})$ & Attendings $(n=18), \%(\mathrm{n})$ & Total $(n=88), \mathrm{p}$ \\
\hline \multicolumn{4}{|l|}{ LT service managed pain well } \\
\hline $34(16)$ & $59(13)$ & $59(10)$ & 0.07 \\
\hline \multicolumn{4}{|l|}{ LT service managed nausea well } \\
\hline Agree $\quad 87(41)$ & $86(19)$ & $82(14)$ & 0.9 \\
\hline \multicolumn{4}{|l|}{ LT identified depression } \\
\hline Agree $21(10)$ & $23(5)$ & $59(10)$ & 0.01 \\
\hline \multicolumn{4}{|l|}{ LT service managed depression well } \\
\hline $26(12)$ & $24(5)$ & $53(9)$ & 0.09 \\
\hline
\end{tabular}

Attendings did not agree with the other providers that depression was identified well in the hospitalized LT population and tended to believe they managed depression well.

Bolded values are statistically significant. 
Table 3. Barriers and Benefits to Palliative Care Consultation on Liver Transplant Service

\begin{tabular}{|c|c|c|c|c|}
\hline & $\begin{array}{c}\text { Nurses } \\
(n=47), \%(\mathrm{n})\end{array}$ & $\begin{array}{c}P G Y 1 \\
(n=23), \%(\mathrm{n})\end{array}$ & $\begin{array}{c}\text { Attendings } \\
(n=18), \%(\mathrm{n})\end{array}$ & $\begin{array}{c}\text { Total } \\
(n=88), \mathrm{p}\end{array}$ \\
\hline \multicolumn{5}{|l|}{ Barriers to palliative care consultation } \\
\hline $\mathrm{PC}$ is always or usually discussed on rounds & $0(0)$ & $0(0)$ & $38(6)$ & $<\mathbf{0 . 0 1}$ \\
\hline Clear criteria for PC consultation are not available & 50 (19) & $42(8)$ & $33(5)$ & 0.5 \\
\hline I requested PC consultation for patient on LT list & $79(37)$ & $91(19)$ & $38(6)$ & $<\mathbf{0 . 0 1}$ \\
\hline \multicolumn{5}{|l|}{ Reasons for failure to consult palliative care } \\
\hline It is difficult to prognosticate end of life in ESLD patients & $39(11)$ & $47(9)$ & $40(6)$ & 0.4 \\
\hline PC and LT are mutually exclusive & $11(4)$ & $11(2)$ & $20(3)$ & 0.6 \\
\hline It is not my place to suggest PC consultation & $55(21)$ & $68(13)$ & $7(1)$ & $<\mathbf{0 . 0 1}$ \\
\hline I did not believe PC would offer additional benefit & $8(3)$ & $32(6)$ & $33(5)$ & $\mathbf{0 . 0 3}$ \\
\hline I thought the patient would perceive team is giving up & $19(7)$ & $21(4)$ & $33(5)$ & 0.5 \\
\hline Involving $\mathrm{PC}$ is giving up on the patient & $5(2)$ & $5(1)$ & $7(1)$ & 1.0 \\
\hline \multicolumn{5}{|l|}{ Benefits to palliative care consultation } \\
\hline Patients welcomed GOC discussions & $84(38)$ & 77 (17) & $87(14)$ & 0.07 \\
\hline Patients were grateful for PC consultation & $90(35)$ & $100(18)$ & $100(15)$ & 0.2 \\
\hline PC helped provide higher quality of care & $93(38)$ & $100(18)$ & $100(15)$ & 0.3 \\
\hline $\begin{array}{l}\text { There were patients who did not receive PC consultation } \\
\text { who would have benefitted from one }\end{array}$ & $93(42)$ & 95 (18) & $50(8)$ & $<\mathbf{0 . 0 1}$ \\
\hline
\end{tabular}

Most providers agree that there are no clear criteria for consulting PC in the LT population. Respondent groups agreed that PC and LT are not mutually exclusive. Nurses and PGY1 did not recommend PC because they felt it was not their place. Respondents agreed that there were multiple benefits to PC consultation. Attendings were least likely to report that there were patients who may have benefitted from PC but did not receive a $\mathrm{PC}$ consultation.

ESLD, end-stage liver disease; GOC, goals of care; PC, palliative care.

Bolded values are statistically significant.

patients (Fig. 1). In addition, attendings were much more likely than nurses and PGY1 to identify the patient and family members as creating a barrier to PCS consultation. While one-third of attendings (38\%) perceived that PCS consultation was usually or always discussed on rounds, no nurses $(0 \%)$ or PGY1 $(0 \%)$ concurred ( $p=0.0001$; Table 3$)$. Many respondents cited lack of clear criteria for involving PCS and difficulty prognosticating end of life in ESLD patients as significant barriers. Furthermore, nurses and PGY1 felt that it was not their place to suggest a consultation. Attendings
$(93 \%, n=82)$ were more likely than PGY1 $(67 \%, n=59)$ and nurses $(55 \%, n=48)$ to describe PCS to their patients as endof-life care $(p=0.03)$. Significantly, few respondents stated that PC and LT were mutually exclusive (Table 3 ).

Many providers reported significant benefits to PCS consultation, although attendings were least likely to perceive PCS as improving quality of life (Fig. 2). Most participants reported that patients and families welcomed GOC discussions and were grateful for PCS consultation (Table 3). Finally, the majority of respondents in all groups agreed that

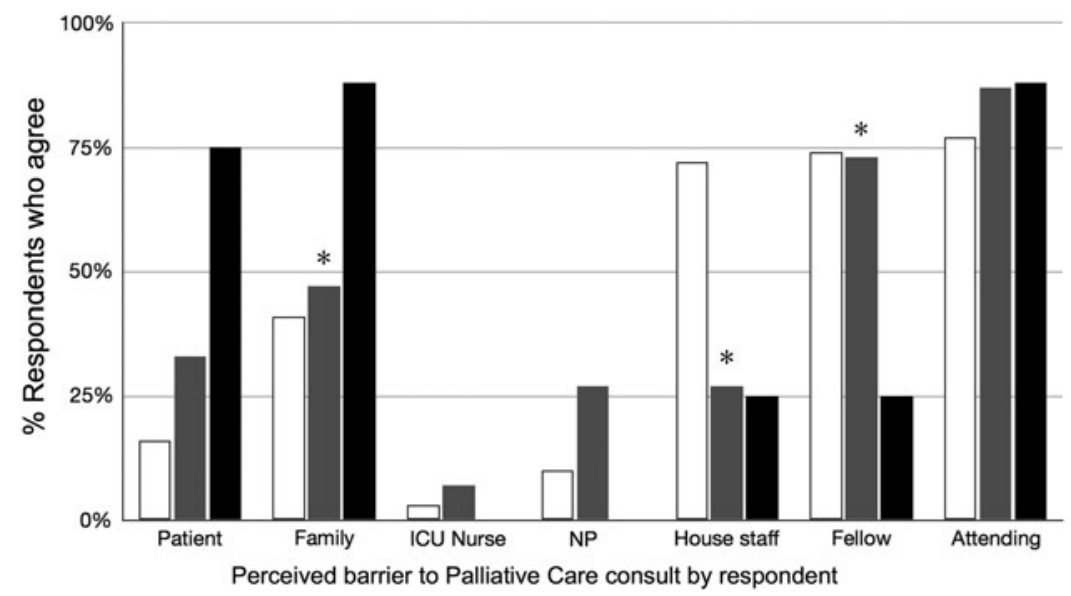

FIG. 1. Perceived barrier to PC consultation by respondent. This figure shows the percentage of respondents by group who perceived patients, families, or groups of healthcare providers as a barrier to PC consultation. Attendings were more likely to perceive the patient and family as a barrier, while nurses were more likely to perceive the house staff as a barrier. All groups (nurses, PGYI, attendings) perceived attendings as a barrier to PC consultation. Nurses are in white, PGYI are in gray, attendings are in solid black. ${ }^{*} p$ Value $<0.05$ representing comparison across all three respondent groups. PC, palliative care. 


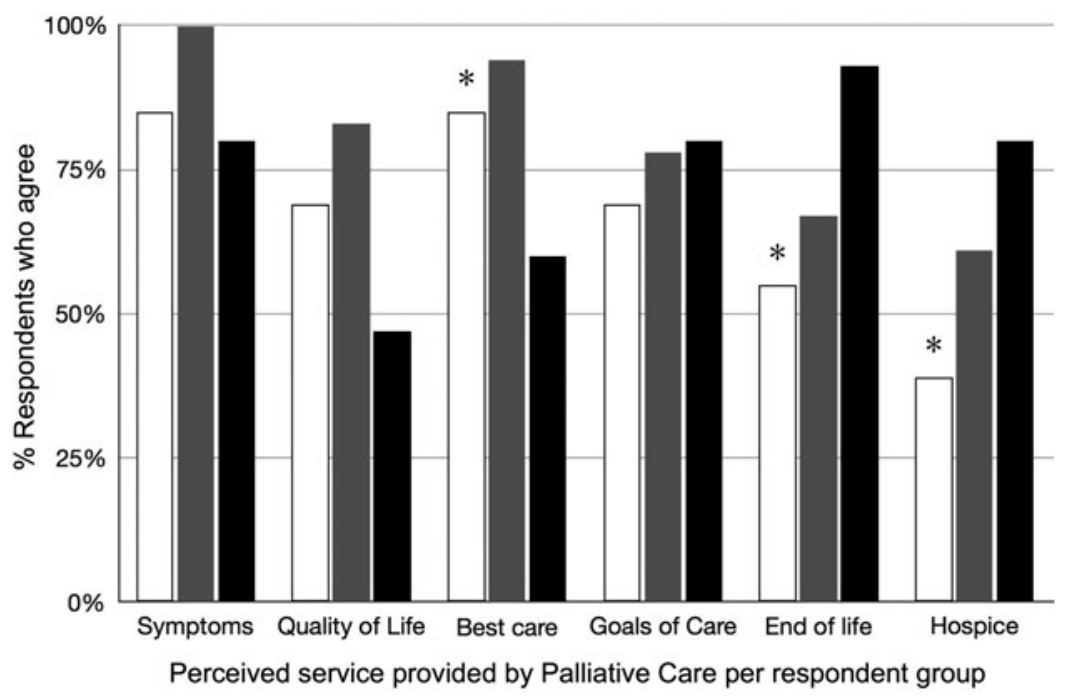

FIG. 2. Attendings equate PC with end-of-life care. This figure shows how respondents present various palliative care services to their patients. Attendings were overwhelmingly more likely to present PC as end-of-life care and hospice compared to nurses and PGYI. Nurses and PGYI were more likely to present PC as part of providing the best quality of care. Nurses are in white, PGYI are in gray, attendings are in solid black. $* p$ Value $<0.05$ representing comparison across all three respondent groups.

PCS helped the LT service provide higher quality of care to their patients (Fig. 2).

\section{Discussion}

Overall, few patients on the LT service were referred for PCS despite the overwhelming recognition of the benefit of PCS by LT clinicians. The main barriers to involving PCS in the care of LT patients appeared to be (1) confusion over optimal timing and referral criteria, (2) describing PCS as end-of-life care, and (3) a lack of open dialogue on rounds, particularly as nurses and PGY1 perceived the discussion of PCS during rounds differently than attendings. Interestingly, all respondents said that the attending physicians were a major barrier to PCS consultations for their patients, including attendings. Despite the barriers identified by our study and the very low rate of PCS consultation among LT patients, clinicians consistently reported that PCS provided benefits to patients and their families by improving the quality of care provided to the patients, and that patients and their families welcomed GOC discussions and were grateful for PCS involvement.

LT clinicians generally agreed that symptom management was not expertly managed on the LT service. Depression is a common symptom in the ESLD population, affecting $14 \%$ of people with cirrhosis in one study, ${ }^{13}$ yet LT providers reported that they did not identify or manage depression well in LT patients. Regarding pain management, valid concerns from all clinicians regarding use of opioid medication in the ESLD and pretransplant population due to the risk of precipitating hepatic encephalopathy, or potentially preventing them from receiving a transplant, appear to contribute to a perception among many clinicians that pain is not well controlled. PCS teams provide expertise in symptom management and could be helpful to LT clinicians and patients in this regard.

The results of this survey indicate that providers do not agree on the optimal timing of GOC discussions and at- tendings are more likely to say that they should be the ones discussing GOC in the clinic rather than primary care providers. Despite an ideal of having these discussions in the outpatient setting, most clinicians perceive that patients are admitted to the hospital not having had these conversations and many clinicians ultimately have these difficult discussions with surrogate decision makers because patients become too ill to participate. The results of this survey suggest that PCS involvement routinely and earlier in the course of ESLD patients could help in elucidating GOC when patients are able to participate in the conversation.

While there are few similar studies of PCS and LT, there were limitations. While our initial sample included $200 \mathrm{cli}-$ nicians, only 88 responded giving a response rate of $44 \%$. This number of respondents limited our power. Nonetheless, we did find statistically significant results; our response rate is acceptable for an anonymous, e-mailed survey, ${ }^{14}$ and we have a broad representation of LT clinicians among respondents. Our study was conducted at one academic medical center, which may limit the generalizability of the findings to other LT units. There may also have been a bias toward putting the LT service in a better light; however, the fact that clinicians admitted to areas where care could be improved and that attendings identified themselves as a barrier to PCS consultation suggests that respondents answered honestly. The fact that we have a well-established, 14-year-old PCS may have biased results toward seeing PCS in a favorable light, although it would be expected that any PCS would provide high-quality care. Finally, we depended on survey responses and were not able to independently verify results such as how often PCS consultation is discussed on rounds. Because we were interested in clinician perceptions, it is not necessary to ascertain the actual rate of such occurrences.

While most studies of PC include people with advanced cancer, there is ample evidence of benefit to people with a broad range of serious illness and their caregivers. Nonetheless, few people with liver disease receive PC, including at 
our institution. Liver disease differs in many ways from terminal cancer in that many liver diseases can be treated or cured and if not, receive an LT. ${ }^{15}$ Despite this possibility, morbidity and mortality are still significant for patients with liver disease who are unable to receive a transplant and in those who are awaiting transplantation. Given this reality for people with liver disease, we recommend that PCS be offered early to patients awaiting LT to enhance the quality of life for these patients during hospitalization. Based on the results of this study, we suggest that PCS expand access to PC for LT patients by establishing clear criteria for referral such as the MELD score or number of hospitalizations, targeting educational interventions about PCS, including shared rounding on LT service, and sharing data from this study that demonstrates that LT providers report benefits to their patients from PC consultation. Given that most large hospitals and academic medical centers offer PCS, collaboration between LT and PCS providers simply requires outreach and discussions rather than establishing new services ${ }^{16,17}$ Future work to help increase access to PC for LT patients includes surveying more LT programs and assessing the impact of PCS on patient outcomes, including through randomized clinical trials of PCS or by incorporating PCS interventions into existing LT studies.

\section{Acknowledgments}

The authors thank the UCSF Liver Center for its support and all of the providers on the Liver Transplant service for their participation. This work was supported, in part, by the UCSF Liver Center (P30 DK026743).

\section{Author Disclosure Statement}

No competing financial interests exist.

\section{References}

1. Scientific Registry of Transplant Recipients/Organ Procurement and Transplantation Network. Annual Data Report: Liver. 2015. www.HRSA.gov (Last accessed December 2015).

2. Kamath PS, Wiesner RH, Malinchoc M, et al.: A model to predict survival in patients with end-stage liver disease. Hepatology 2001;33:464-470.

3. Volk ML, Tocco RS, Bazick J, et al.: Hospital readmissions among patients with decompensated cirrhosis. Am J Gastroenterol 2012;107:247-252.

4. D’Amico G, Garcia-Tsao G, Pagliaro L. Natural history and prognostic indicators of survival in cirrhosis: A systematic review of 118 studies. J Hepatol 2006;1:217-231.
5. Morrison RS. Health care system factors affecting end-oflife care. J Palliat Med 2005;8(Suppl 1):S79-S87.

6. El-Jawahri A, Greer JA, Temel JS. Does palliative care improve outcomes for patients with incurable illness? A review of the evidence. J Support Oncol 2011;9:87-94.

7. Temel JS, Greer JA, Muzikansky A, et al.: Early palliative care for patients with metastatic non-small cell lung cancer. New Engl J Med 2010;363:733-742.

8. Wright AA, Zhang B, Ray A, et al.: Associations between end-of-life discussions, patient mental health, medical care near death, and caregiver bereavement adjustment. JAMA 2008;300:1665-1673.

9. Larson AM, Curtis JR. Integrating palliative care for liver transplant candidates: "Too well for transplant, too sick for life.” JAMA 2006;295:2168-2176.

10. Poonja Z, Brisebois A, van Zanten SV, et al.: Patients with cirrhosis and denied liver transplants rarely receive adequate palliative care or appropriate management. Clin Gastroenterol Hepatol 2014;12:692-698.

11. Devillis, RF: Scale Development: Theory and Applications, 3rd ed. Thousand Oaks, CA: Sage Publications, 2012.

12. IBM SPSS Statistics for Macintosh, Version 22.0. Armonk, NY: IBM Corp, 2013.

13. Kalaitzakis E, Josefsson A, Bjornsson E. Psychological distress in patients with liver cirrhosis. Gastroenterology 2008;134:A625.

14. Kellerman S, Herold J. Physician response to surveys: A review of the literature. Am J Prev Med 2001;20:61-67.

15. Boyd K, Kimbell B, Murray S, Iredale J. Living and dying well with end-stage liver disease: Time for palliative care? Hepatology 2012;55:1650-1651.

16. Goldsmith B, Dietrich J, Du Q, Morrison RS. Variability in access to hospital palliative care in the United States. J Palliat Med 2008;11:1094-1102.

17. Pantilat SZ, Kerr KM, Billings JA, et al.: Palliative Care Services in California Hospitals: Program Prevalence and Hospital Characteristics. J Pain Symptom Manage 2012; 43:39-46.
Address correspondence to: Kendall R. Beck, MD Division of Gastroenterology Department of Medicine University of California San Francisco 505 Parnassus Avenue S357 San Francisco, CA 94143

E-mail: kendall.beck@ucsf.edu 